\title{
Consensus Analysis of Fractional-Order Multiagent Systems with Double-Integrator
}

\author{
Chunde Yang, Wenjing Li, and Wei Zhu \\ Research Center of System Theory and Application, Chongqing University of Posts and Telecommunications, Chongqing 400065, China \\ Correspondence should be addressed to Wei Zhu; zhuwei@cqupt.edu.cn
}

Received 25 July 2016; Accepted 14 December 2016; Published 2 January 2017

Academic Editor: Seenith Sivasundaram

Copyright (C) 2017 Chunde Yang et al. This is an open access article distributed under the Creative Commons Attribution License, which permits unrestricted use, distribution, and reproduction in any medium, provided the original work is properly cited.

\begin{abstract}
In nature, many phenomena can be explained by coordinated behavior of agents with fractional-order dynamics. In this paper, the consensus problem of fractional-order multiagent systems with double-integrator is studied, where the fractional-order satisfies $0<\alpha<2$. Based on the fractional-order stability theory, Mittag-Leffler function, and Laplace transform, a necessary and sufficient condition is obtained under the assumption that the directed graph for the communication network contains a directed spanning tree. Finally, an example with simulation is presented to illustrate the theoretical results.
\end{abstract}

\section{Introduction}

The last few decades have witnessed considerable progress in the study of multiagent systems due to its wide applications. Typical research in this field includes the problems of consensus [1], formation control [2], cooperative control of unmanned vehicle [3], flocking [4], deployment [5], and rendezvous [6]. One can refer to many references therein for more details about these topics.

Note that many investigations focused on the multiagent systems with integer-order dynamics. In fact, many practical engineering systems cannot be well explained in the framework of integer-order dynamics, such as electromagnetic waves, the heat flux of the thermal field of the furnace wall, food seeking of microbes, and underwater vehicles operating in lentic lakes $[7,8]$. Furthermore, it was pointed out in $[9,10]$ that the well-studied integer-order systems are just the special cases of fractional-order ones. From an implementation point of view, multiagent systems with fractional-order dynamics are an important issue.

In recent years, the consensus problem of fractionalorder multiagent systems has become a hot topic. Distributed coordination control of fractional-order multiagent systems was early discussed by Cao and Ren in [11-13]. Based on the stability theory of fractional-order systems, Song and Cao [14] investigated the consensus of the fractional-order linear multiagent systems (MAS) with directed interaction topology in terms of linear matrix inequalities (LMIs). By using algebraic graph theory and Lyapunov method, the leaderfollowing consensus problem of fractional-order multiagent systems with nonlinear dynamics was considered in [15]. Based on the stability theory of fractional-order differential systems and matrix inequalities, some consensus criteria were obtained in [16] on leader-following consensus problem. In [17], based on the Laplace transform and frequency domain theory of the fractional-order operator, the consensus of delayed multiagent systems with directed weighted topologies was studied. In [18], the authors discussed the consensus of fractional-order heterogeneous multiagent systems by the Kronecker product technique, the fractional-order stability theory, and LMIs. In [19], the Mittag-Leffler stability and the fractional Lyapunov direct method were firstly introduced into the fractional multiagent systems. Sufficient conditions were given to guarantee that the leader-following consensus can be achieved with both single-integrator dynamics and double-integrator dynamics, respectively. In [20], the robust consensus problem of fractional-order linear multiagent systems is discussed. Some new LMI-based sufficient conditions were obtained.

With the above considerations, in this paper, the consensus of fractional-order multiagent systems with doubleintegrator and fractional-order $\alpha$ satisfying $\alpha \in(0,2)$ over 
a directed communication graph is investigated. Compared with most of existing literature, not only is fractional-order $\alpha$ satisfying $0<\alpha \leq 1$ discussed, but also $1<\alpha<2$ is studied. A novel control protocol with less information of neighboring agents is proposed, which may save computation and online resources. Furthermore, not only is the sufficient condition on consensus given, but also the necessary condition is presented.

The rest of this paper is organized as follows. Section 2 describes some preliminary results of graph theory and fractional-order systems. The main results are derived in Section 3. One simulation example is given in Section 4 to illustrate the effectiveness of the theoretical results. Conclusions are drawn in Section 5.

\section{Preliminaries}

2.1. Graph Theory. For a system with $n$ agents, the communication graph for all agents can be modeled by a directed graph $\mathscr{G}=(\mathscr{V}, \mathscr{E})$, where $\mathscr{V}=\left\{v_{1}, v_{2}, \ldots, v_{n}\right\}$, and $\mathscr{E} \subseteq \mathscr{V} \times \mathscr{V}$ are the sets of vertices and edges of the graph $G$, respectively. Each edge can be denoted as $\left(v_{i}, v_{j}\right)$ which means agent $v_{j}$ can access the information of agent $v_{i}$. Accordingly, agent $v_{i}$ is a neighbor of agent $v_{j}$. All neighbors of agent $i$ are denoted by $\mathcal{N}_{i}=\left\{v_{j} \in \mathscr{V}:\left(v_{j}, v_{i}\right) \in \mathscr{E}\right\}$. A directed path is a sequence of edges in a directed graph with the form $\left(v_{1}, v_{2}\right),\left(v_{2}, v_{3}\right), \ldots$, where $v_{i} \in \mathscr{V}$. A directed graph has a directed spanning tree if there exists at least one agent that has a directed path to all other agents. In a digraph $\mathscr{G}$, if $\mathcal{U}$ is a nonempty subset of $\mathscr{V}$ and $u \nrightarrow v$ for all $u \in \mathscr{U}$ and $v \in \mathscr{V}-\mathscr{U}$, then $\mathcal{U}$ is said to be closed.

The communication graph can be represented by two types of matrices: the adjacency matrix $A=\left[a_{i j}\right] \in R^{n \times n}$ with $a_{i j}>0$ if $\left(v_{j}, v_{i}\right) \in \mathscr{E}$ and $a_{i j}=0$ otherwise. $D=$ $\operatorname{diag}\left\{d_{1}, d_{2}, \ldots, d_{n}\right\}$ is the degree matrix whose diagonal elements are defined by $d_{i}=\sum_{j \in \mathcal{N}_{i}, j \neq i} a_{i j}$. The Laplace matrix $L=\left[l_{i j}\right] \in R^{n \times n}$ with $l_{i i}=\sum_{j \in \mathcal{N}_{i}, j \neq i} a_{i j}$ and $l_{i j}=-a_{i j}, i \neq j$, and $L=D-A$.

2.2. Caputo Fractional Derivative. The Caputo fractional derivative is defined as follows:

$$
{ }_{a}^{C} D_{t}^{\alpha} f(t)=\frac{1}{\Gamma(n-\alpha)} \int_{a}^{t} \frac{f^{(n)}(\tau)}{(t-\tau)^{\alpha-n+1}} d \tau,
$$

$$
n-1<\alpha \leq n
$$

where $\alpha$ is the fractional-order and $\Gamma(\cdot)$ is the Gamma function defined as $\Gamma(\tau)=\int_{0}^{\infty} t^{\tau-1} e^{-t} d t$. A simple notion $D^{\alpha} f(t)$ will be used to denote ${ }_{a}^{C} D_{t}^{\alpha} f(t)$.

Let $\mathscr{L}\{\cdot\}$ denote the Laplace transform of a function; then we have

$$
\begin{aligned}
\mathscr{L} & \left\{D^{\alpha} f(t)\right\} \\
& = \begin{cases}s^{\alpha} F(s)-s^{\alpha-1} f(0+), & \alpha \in(0,1] \\
s^{\alpha} F(s)-s^{\alpha-1} f(0+)-s^{\alpha-2} \dot{f}(0+), & \alpha \in(1,2),\end{cases}
\end{aligned}
$$

where $f(0+)$ and $\dot{f}(0+)$ denote the right limit of $f(t)$ and $\dot{f}(t)$ at 0 , respectively.

Definition 1 (see [21]). The two-parameter function of the Mittag-Leffler type is defined by

$$
E_{\alpha, \beta}(z)=\sum_{k=0}^{\infty} \frac{z^{k}}{\Gamma(k \alpha+\beta)},
$$

where $\alpha>0, \beta>0$, and $z$ is a complex number.

Particularly, when $\beta=1$, write $E_{\alpha, 1}(z) \triangleq E_{\alpha}(z)=$ $\sum_{k=0}^{\infty}\left(z^{k} / \Gamma(k \alpha+1)\right)$.

Moreover, the Laplace transform of the Mittag-Leffler function in two parameters is

$$
\mathscr{L}\left\{t^{\beta-1} E_{\alpha, \beta}\left(-\lambda t^{\alpha}\right)\right\}=\frac{s^{\alpha-\beta}}{s^{\alpha}+\lambda}, \quad\left(R(s)>|\lambda|^{1 / \alpha}\right) .
$$

2.3. System Model Description. Consider the following fractional-order double-integrator multiagent systems with $n$ agents:

$$
\begin{aligned}
& D^{\alpha} x_{i}(t)=v_{i}(t) \\
& D^{\alpha} v_{i}(t)=u_{i}(t), \\
& \quad t \geq 0, \quad i \in T,
\end{aligned}
$$

where $0<\alpha<2, T=\{1,2, \ldots, n\}, x_{i}(t) \in R$, and $v_{i}(t) \in R$ are the states and $u_{i}(t) \in R$ is the control input of the $i$ th agent, respectively.

Definition 2. For multiagent systems (5), the protocols $u_{i}(t)$ are said to solve the consensus if and only if, for any initial states, the states of the agents satisfy

$$
\begin{aligned}
\lim _{t \rightarrow \infty}\left\|x_{j}(t)-x_{i}(t)\right\| & =0, \quad i, j \in T, \\
\lim _{t \rightarrow \infty}\left\|D^{\alpha} x_{j}(t)-D^{\alpha} x_{i}(t)\right\| & =0, \quad i, j \in T .
\end{aligned}
$$

To study the consensus of multiagent systems (5), the following consensus control protocol will be used:

$$
u_{i}(t)=-\sum_{j \in \mathcal{N}_{i}, j \neq i} a_{i j}\left(x_{i}(t)-x_{j}(t)\right)-k D^{\alpha} x_{i}(t),
$$

$i \in T$,

where $k>0$ is the control parameter to be designed later.

Let $x(t)=\left(x_{1}(t), x_{2}(t), \ldots, x_{n}(t)\right)^{T}$ and $v(t)=\left(v_{1}(t)\right.$, $\left.v_{2}(t), \ldots, v_{n}(t)\right)^{T}$; then (5) can be rewritten in the following compact form:

$$
\begin{aligned}
& D^{\alpha} x(t)=v(t) \\
& D^{\alpha} v(t)=-L x(t)-k v(t) .
\end{aligned}
$$

Denote $\mathbf{1}_{n-1}^{T}=\underbrace{[1, \ldots, 1]}_{n-1}, \tilde{x}(t)=S x(t)$, and $\widetilde{v}(t)=S v(t)$, where

$$
S=\left(\begin{array}{cc}
1 & 0 \\
-\mathbf{1}_{n-1} & I_{n-1}
\end{array}\right) \text {. }
$$


Then, by (8), we have

$$
\begin{aligned}
& D^{\alpha} \tilde{x}(t)=\widetilde{v}(t) \\
& D^{\alpha} \widetilde{v}(t)=-\left(\mathrm{SLS}^{-1}\right) \widetilde{x}(t)-k \widetilde{v}(t) .
\end{aligned}
$$

Define $\widehat{x}_{1}(t)=x_{1}(t), \hat{x}_{2}(t)=\left(x_{2}(t)-x_{1}(t), \ldots, x_{n}(t)-x_{1}(t)\right)^{T}$, $\widehat{v}_{1}(t)=v_{1}(t)$, and $\widehat{v}_{2}(t)=\left(v_{2}(t)-v_{1}(t), \ldots, v_{n}(t)-v_{1}(t)\right)^{T}$. Then, (10) can be decoupled as follows:

$$
\begin{aligned}
& D^{\alpha} \widehat{x}_{2}(t)=\widehat{v}_{2}(t) \\
& D^{\alpha} \widehat{v}_{2}(t)=-\widetilde{L}_{2}(t)-k \widehat{v}_{2}(t),
\end{aligned}
$$

where

$$
\widetilde{L}=\left(\begin{array}{cccc}
d_{2}+a_{12} & a_{13}-a_{23} & \cdots & a_{1 n}-a_{2 n} \\
a_{12}-a_{32} & d_{3}+a_{13} & \cdots & a_{1 n}-a_{3 n} \\
\vdots & \vdots & \ddots & \vdots \\
a_{12}-a_{n 2} & a_{13}-a_{n 3} & \cdots & d_{n}+a_{1 n}
\end{array}\right)
$$

Let $Y(t)=\left(\widehat{x}_{2}^{T}(t), \widehat{v}_{2}^{T}(t)\right)^{T}$; (11) can be rewritten in the following compact form:

$$
D^{\alpha} Y(t)=P Y(t)
$$

where

$$
P=\left(\begin{array}{cc}
0 & I_{n-1} \\
-\widetilde{L} & -k I_{n-1}
\end{array}\right) \text {. }
$$

\section{Main Results}

It will be found that the following lemmas are useful to prove the necessary and sufficient condition given in this section.

Lemma 3. All the eigenvalues of matrix $\widetilde{L}$ have positive real parts if and only if the communication graph of all agents has a directed spanning tree.

Proof. By simple calculation, we have

$$
\mathrm{SLS}^{-1}=\left(\begin{array}{cc}
0 & L_{1} \\
\mathbf{0}_{n-1}^{T} & \widetilde{L}
\end{array}\right),
$$

where $\mathbf{0}_{n-1}=\underbrace{[0, \ldots, 0]}_{n-1}$ and $L_{1}=\left[-a_{12}, \ldots,-a_{1 n}\right]$. According to [22], $L$ has exactly one zero eigenvalue and all the other eigenvalues have positive real parts if and only if the communication graph of all agents has a directed spanning tree. Then, it follows from (15) that the conclusion of Lemma 3 holds.

Lemma 4 (see [23]). Let

$$
Q=\left(\begin{array}{cc}
0 & I \\
-H & -k I
\end{array}\right)
$$

Assume that the control parameter $k$ satisfies $k^{2}>$ $\left(\max _{\mu \in \rho(H)}\{|\operatorname{Im} \mu|\}\right)^{2} / \min _{\mu \in \rho(H)} \operatorname{Re} \mu,\left(\min _{\mu \in \rho(H)} \operatorname{Re} \mu \neq 0\right)$, where $\rho(H)$ denotes the set of all eigenvalues of $H$. Then $\max _{\theta \in \rho(\mathrm{Q})} \operatorname{Re} \theta<0$ if and only if $H$ is positive stable (i.e., all eigenvalues have positive real parts).

Lemma 5 (see [24]). Consider the general fractional-order linear differential system:

$$
D^{\alpha} x(t)=A x(t),
$$

where $0<\alpha<2, x(t) \in R^{n}$, and $A \in R^{n \times n}$. System (17) is asymptotically stable if and only if $\left|\arg \left(\lambda_{i}\right)\right|>\alpha \pi / 2$, for $i=$ $1, \ldots, n$, and $\lambda_{i}$ is any eigenvalue of coefficient matrix $A$.

By Lemma 2.2 in [25], with a mild revision, we have the following result.

Lemma 6. If $A$ is a Hurwitz matrix, then there are some real constants $K \geq 1$ and $\lambda>0$ such that

$$
\left\|E_{\alpha, \beta}\left(A t^{\alpha}\right)\right\| \leq\left\|e^{A t^{\alpha}}\right\| \leq K e^{-\lambda t},
$$

for any $\alpha \geq 1$ and $\beta>0$.

Lemma 7 (see [26]). A digraph $G=(\mathscr{V}, \mathscr{E})$, with $|\mathscr{V}| \geqslant 2$, has no directed spanning tree if and only if it has at least two disjoint closed subsets of $\mathscr{V}$.

Now, we are in the position to state the consensus result.

Theorem 8. Consider the fractional-order double-integrator multiagent systems (5) with control protocol (7) and take $k^{2}>$ $\left(\max _{\mu \in \rho(\widetilde{L})}\{|\operatorname{Im} \mu|\}\right)^{2} / \min _{\mu \in \rho(\widetilde{L})} \operatorname{Re} \mu$. Then, the consensus can be achieved if and only if the communication graph of all agents has a directed spanning tree.

Proof (sufficiency). Since the communication graph of all agents has a directed spanning tree, by Lemma 3, all the eigenvalues of matrix $\widetilde{L}$ have positive real parts. It follows from the choice of parameter $k$ and Lemma 4 that $\max _{\theta \in \rho(P)} \operatorname{Re} \theta<0$. Hence, $|\arg (\theta)|>\pi / 2 \geq \alpha \pi / 2$ for $\alpha \in(0,1]$. It follows from Lemma 5 that the consensus can be achieved asymptotically for $\alpha \in(0,1]$.

For $\alpha \in(1,2)$, taking the Laplace transform on (13), we have

$$
\mathscr{L}\{Y(t)\}=\frac{s^{\alpha-1} Y(0+)+s^{\alpha-2} \dot{Y}(0+)}{I s^{\alpha}-P} .
$$

Then, taking the inverse Laplace transform of (19), one can obtain that

$$
Y(t)=E_{\alpha}\left(P t^{\alpha}\right) Y(0+)+t E_{\alpha, 2}\left(P t^{\alpha}\right) \dot{Y}(0+) .
$$

In addition, since $P$ is a Hurwitz matrix, it follows from Lemma 6 that there exist constants $K_{1}, K_{2}>1$ and $\lambda_{1}>0$ and $\lambda_{2}>0$ such that

$$
\begin{aligned}
\|Y(t)\| & \leq\left\|E_{\alpha}\left(P t^{\alpha}\right) Y(0+)\right\|+\left\|t E_{\alpha, 2}\left(P t^{\alpha}\right) \dot{Y}(0+)\right\| \\
& \leq K_{1} e^{-\lambda_{1} t}\|Y(0+)\|+t K_{2} e^{-\lambda_{2} t}\|\dot{Y}(0+)\| .
\end{aligned}
$$


Therefore, $\|Y(t)\| \rightarrow 0$ as $t \rightarrow \infty$ for any initial condition, which implies that the consensus can be achieved asymptotically for $\alpha \in(1,2)$.

Thus, the consensus of fractional-order double-integrator systems (5) with $0<\alpha<2$ can be achieved asymptotically if the communication graph of all agents has a directed spanning tree.

(Necessity). The consensus of fractional-order doubleintegrator multiagent systems (5) can be achieved, which implies that $x_{i} \rightarrow x_{j}$ and $D^{\alpha} x_{i} \rightarrow D^{\alpha} x_{j}$ as $t \rightarrow \infty$ for any initial states.

Suppose that the communication graph of all agents has no directed spanning tree. Then, it follows from Lemma 7 that there are at least two disjoint closed sets of nodes in graph $\mathscr{G}$. Without loss of generality, we consider the following special case; that is, there are exactly two disjoint closed sets in graph $\mathscr{G}$, say $\mathscr{V}_{1}=\left\{v_{1}\right\}$ and $\mathscr{V}_{2}=\left\{v_{2}\right\}$; that is, there is only one node in $\mathscr{V}_{1}$ and $\mathscr{V}_{2}$, respectively. If there are more disjoint closed sets or there are more nodes in each disjoint closed sets, it can be proved by a similar argument only with more complex computation.

For $\alpha \in(0,1]$, given the initial conditions satisfying $x_{1}(0)=v_{1}(0)=c_{1}$ and $x_{2}(0)=v_{2}(0)=c_{2}$, where $c_{1}$ and $c_{2}$ are any given constants, by a direct computation, we have $x_{1}(t)=c_{1} \mathscr{L}^{-1}\left\{\left(s^{2 \alpha-1}+k s^{\alpha-1}+s^{\alpha-1}\right) /\left(s^{2 \alpha}+k s^{\alpha}+1\right)\right\}$, $D^{\alpha} x_{1}(t)=c_{1} \mathscr{L}^{-1}\left\{\left(s^{2 \alpha-1}-s^{\alpha-1}\right) /\left(s^{2 \alpha}+k s^{\alpha}+1\right)\right\}, x_{2}(t)=$ $c_{2} \mathscr{L}^{-1}\left\{\left(s^{2 \alpha-1}+k s^{\alpha-1}+s^{\alpha-1}\right) /\left(s^{2 \alpha}+k s^{\alpha}+1\right)\right\}$, and $D^{\alpha} x_{2}(t)=$ $c_{2} \mathscr{L}^{-1}\left\{\left(s^{2 \alpha-1}-s^{\alpha-1}\right) /\left(s^{2 \alpha}+k s^{\alpha}+1\right)\right\}$. Hence, if $c_{1} \neq c_{2}$, the consensus cannot be reached, which is a contradiction.

For $\alpha \in(1,2)$, given the initial conditions satisfying $x_{1}(0)=v_{1}(0)=c_{1}, x_{2}(0)=v_{2}(0)=c_{2}, \dot{x}_{1}(0)=\dot{v}_{1}(0)=c_{3}$, and $\dot{x}_{2}(0)=\dot{v}_{2}(0)=c_{4}$, where $c_{1}, c_{2}, c_{3}$, and $c_{4}$ are any given constants, by a direct computation, we have $x_{1}(t)=$ $c_{1} \mathscr{L}^{-1}\left\{\left(s^{2 \alpha-1}+k s^{\alpha-1}+s^{\alpha-1}\right) /\left(s^{2 \alpha}+k s^{\alpha}+1\right)\right\}+c_{3} \mathscr{L}^{-1}\left\{\left(s^{2 \alpha-1}+\right.\right.$ $\left.\left.k s^{\alpha-1}+s^{\alpha-1}\right) /\left(s^{2 \alpha+1}+k s^{\alpha+1}+s\right)\right\}, D^{\alpha} x_{1}(t)=c_{1} \mathscr{L}^{-1}\left\{\left(s^{2 \alpha-1}-\right.\right.$ $\left.\left.s^{\alpha-1}\right) /\left(s^{2 \alpha}+k s^{\alpha}+1\right)\right\}+c_{3} \mathscr{L}^{-1}\left\{\left(s^{2 \alpha-1}-s^{\alpha-1}\right) /\left(s^{2 \alpha+1}+k s^{\alpha+1}+s\right)\right\}$, $x_{2}(t)=c_{2} \mathscr{L}^{-1}\left\{\left(s^{2 \alpha-1}+k s^{\alpha-1}+s^{\alpha-1}\right) /\left(s^{2 \alpha}+k s^{\alpha}+1\right)\right\}+$ $c_{4} \mathscr{L}^{-1}\left\{\left(s^{2 \alpha-1}+k s^{\alpha-1}+s^{\alpha-1}\right) /\left(s^{2 \alpha+1}+k s^{\alpha+1}+s\right)\right\}$, and $D^{\alpha} x_{2}(t)=$ $c_{2} \mathscr{L}^{-1}\left\{\left(s^{2 \alpha-1}-s^{\alpha-1}\right) /\left(s^{2 \alpha}+k s^{\alpha}+1\right)\right\}+c_{4} \mathscr{L}^{-1}\left\{\left(s^{2 \alpha-1}-\right.\right.$ $\left.\left.s^{\alpha-1}\right) /\left(s^{2 \alpha+1}+k s^{\alpha+1}+s\right)\right\}$. Hence, the consensus cannot be reached if $c_{1} \neq c_{2}$ or $c_{3} \neq c_{4}$, which is a contradiction.

The contradiction shows that there must be a directed spanning tree in the communication graph of all agents.

Remark 9. The consensus result about fractional-order multiagent system with double-integrator is presented in Theorem 8 , where not only is fractional-order $\alpha$ satisfying $0<\alpha \leq$ 1 discussed, but also $1<\alpha<2$ is studied. Furthermore, not only is the sufficient condition on consensus given, but also the necessary condition is presented.

\section{A Numerical Example and Simulation}

In this section, a simulation example is presented to illustrate the theoretical results for $0<\alpha \leq 1$ and $1<\alpha<$ 2 , respectively. Simulation comparison with some existing protocols in literature is done, which shows the effectiveness

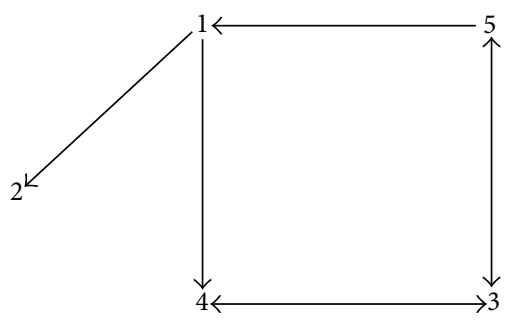

FIGURE 1: Directed communication graph of five agents.

of the Theorem 8. For the sake of comparison, we restate the control protocol without leader in [19] as follows:

$$
\begin{aligned}
& u_{i}(t)=-\sum_{j \in \mathscr{N}_{i}, j \neq i} a_{i j}\left[\left(x_{i}(t)-x_{j}(t)\right)\right. \\
& \left.+\beta\left(D^{\alpha} x_{i}(t)-D^{\alpha} x_{j}(t)\right)\right], \quad i \in T,
\end{aligned}
$$

with $\beta \geq 2 / \sqrt{\min \left\{\left|\lambda_{i}(\widetilde{L})\right|\right\}}$.

Example 1. Consider a directed communication graph with 5 agents given by Figure 1. Assume that the digraph in this figure has $0-1$ weights for simplicity. Here, if agent $j$ is a neighbor of agent $i, a_{i j}=1$; otherwise, $a_{i j}=0$. Hence, the adjacent matrix

$$
A=\left(\begin{array}{lllll}
0 & 0 & 0 & 0 & 1 \\
1 & 0 & 0 & 0 & 0 \\
0 & 0 & 0 & 1 & 1 \\
1 & 0 & 1 & 0 & 0 \\
0 & 0 & 1 & 0 & 0
\end{array}\right)
$$

Then, the Laplacian $L$ and $\widetilde{L}$ are as follows:

$$
\begin{aligned}
L & =\left(\begin{array}{ccccc}
1 & 0 & 0 & 0 & -1 \\
-1 & 1 & 0 & 0 & 0 \\
0 & 0 & 2 & -1 & -1 \\
-1 & 0 & -1 & 2 & 0 \\
0 & 0 & -1 & 0 & 1
\end{array}\right) \\
\widetilde{L} & =\left(\begin{array}{cccc}
1 & 0 & 0 & 1 \\
0 & 2 & -1 & 0 \\
0 & -1 & 2 & 1 \\
0 & -1 & 0 & 2
\end{array}\right)
\end{aligned}
$$

By simple computation, we have $\rho(\widetilde{L})=1,3.3247$, $1.3376+0.5623 i$, and $1.3376-0.5623 i$, where $i^{2}=-1$, and then $k^{2}>0.3162$. Obviously, the communication graph has a directed spanning tree. Thus, the consensus can be achieved for any initial states by Theorem 8 .

In the following, the simulations are done for some $\alpha \epsilon$ $(0,1]$ and $\alpha \in(1,2)$, respectively. 

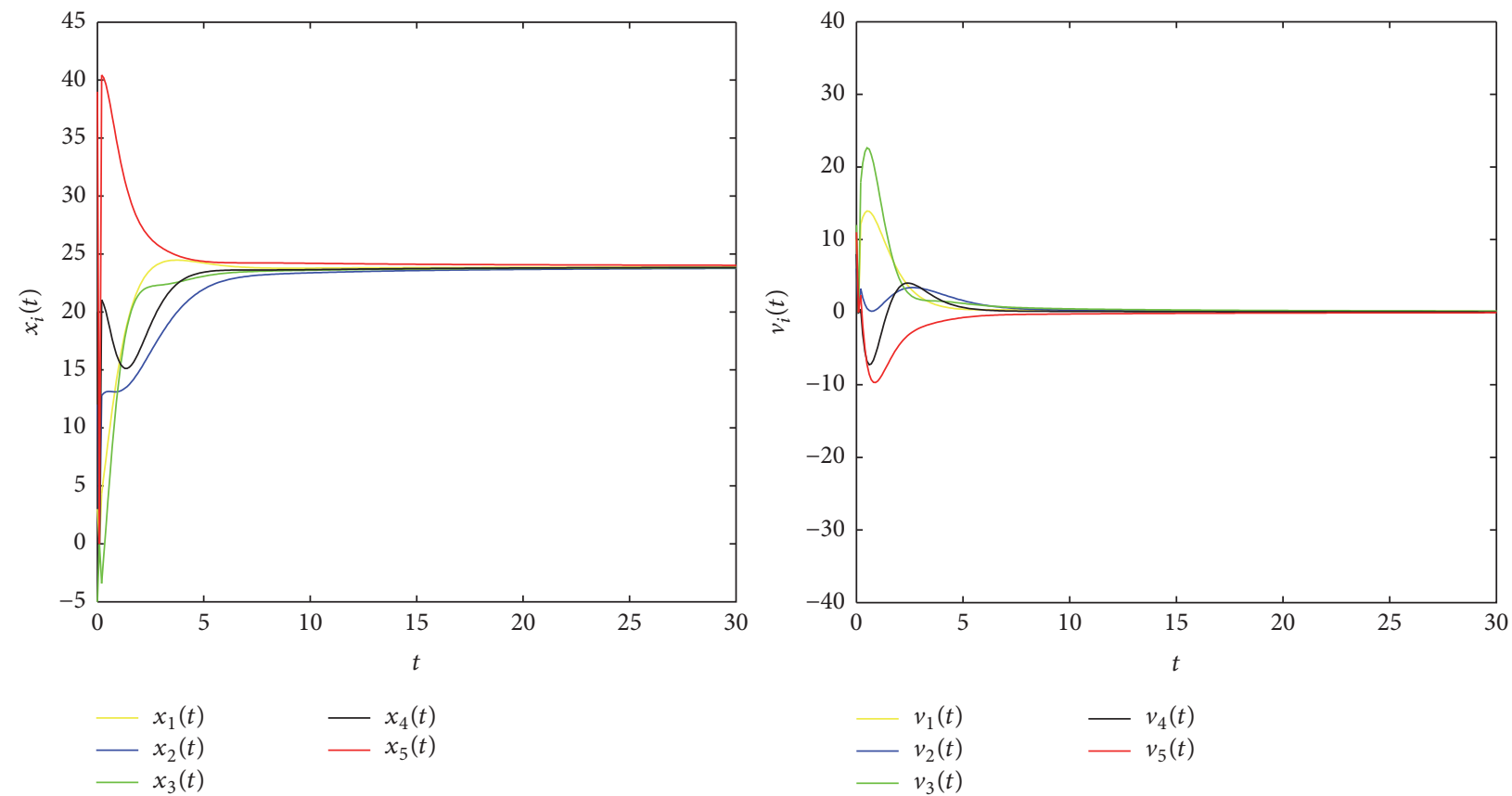

FIGURE 2: $x_{i}(t)$ and $v_{i}(t)$ of each agent with protocol (7), $\alpha=0.9$.
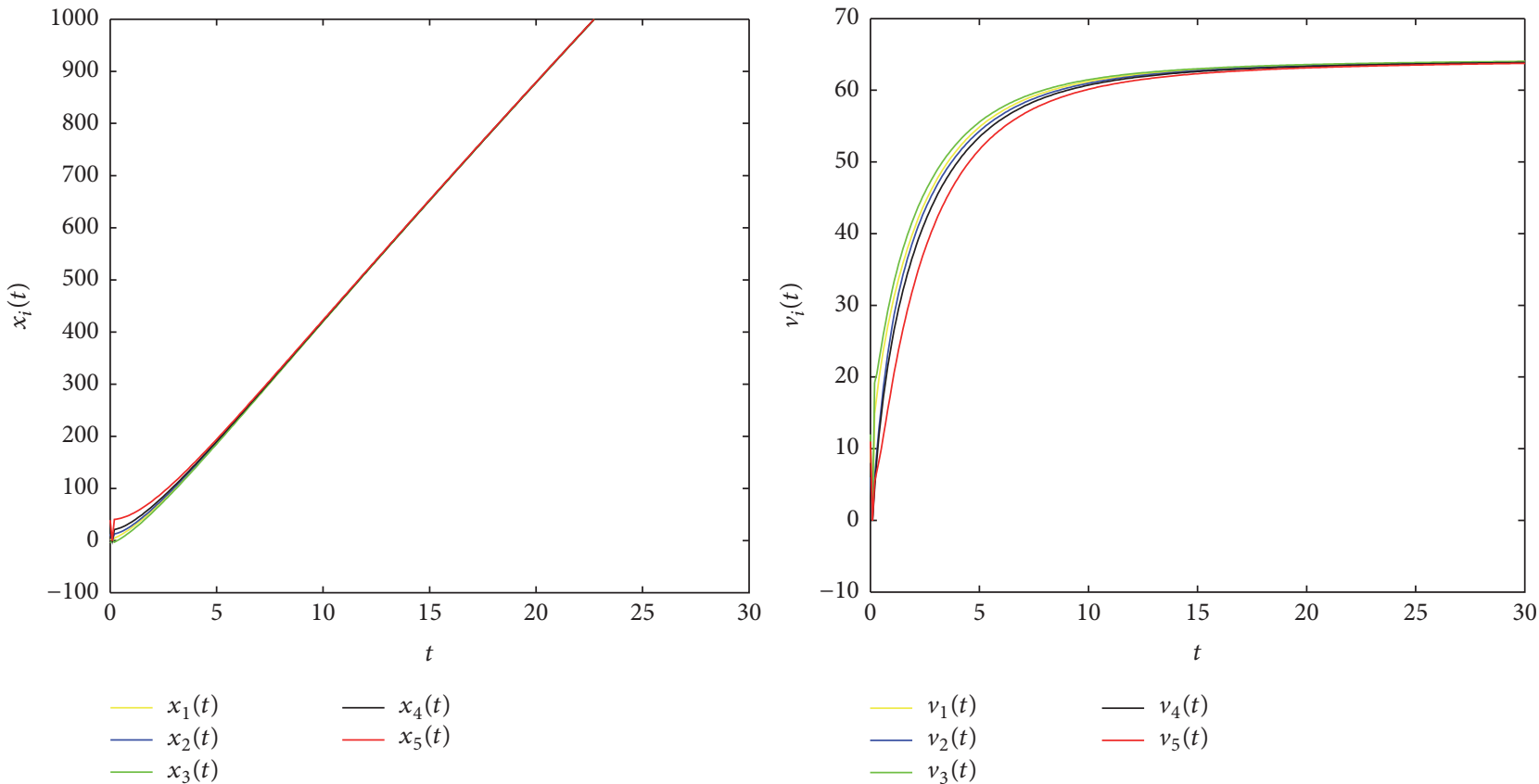

FIGURE 3: $x_{i}(t)$ and $v_{i}(t)$ of each agent with protocol (22), $\alpha=0.9$.

Case 1. $\alpha=0.9, k=2$, and $\beta=2.4$. Assume the initial states for each agent are given as $x_{1}(0)=3, x_{2}(0)=12, x_{3}(0)=$ $-5, x_{4}(0)=20, x_{5}(0)=39, v_{1}(0)=10, v_{2}(0)=6, v_{3}(0)=$ $12, v_{4}(0)=8$, and $v_{5}(0)=11$. The states $x_{i}(t)$ and $v_{i}(t)$ of each agent are depicted in Figures 2 and 3, respectively, which shows the consensus can be achieved asymptotically.
Case 2. $\alpha=1.3, k=2$, and $\beta=2.4$. Further assume that $\dot{x}_{1}(0)=0.2, \dot{x}_{2}(0)=1, \dot{x}_{3}(0)=0.5, \dot{x}_{4}(0)=0.1, \dot{x}_{5}(0)=2$, $\dot{v}_{1}(0)=4, \dot{v}_{2}(0)=0.1, \dot{v}_{3}(0)=0.8, \dot{v}_{4}(0)=3$, and $\dot{v}_{5}(0)=$ 0.2 . The states $x_{i}(t)$ and $v_{i}(t)$ of each agent are depicted in Figures 4 and 5, respectively, which shows the consensus can be achieved asymptotically. 

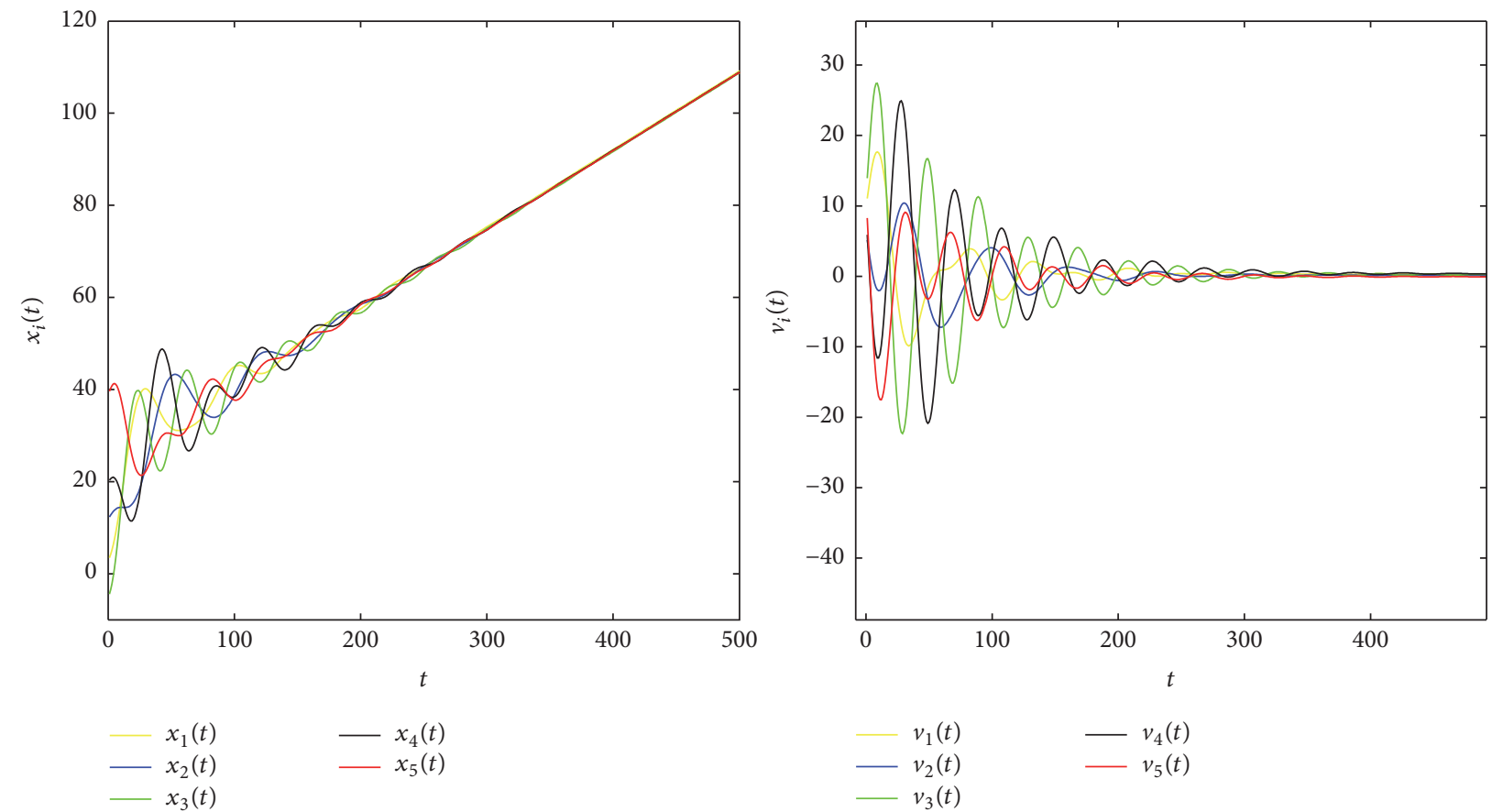

FIGURE 4: $x_{i}(t)$ and $v_{i}(t)$ of each agent with protocol (7), $\alpha=1.3$.
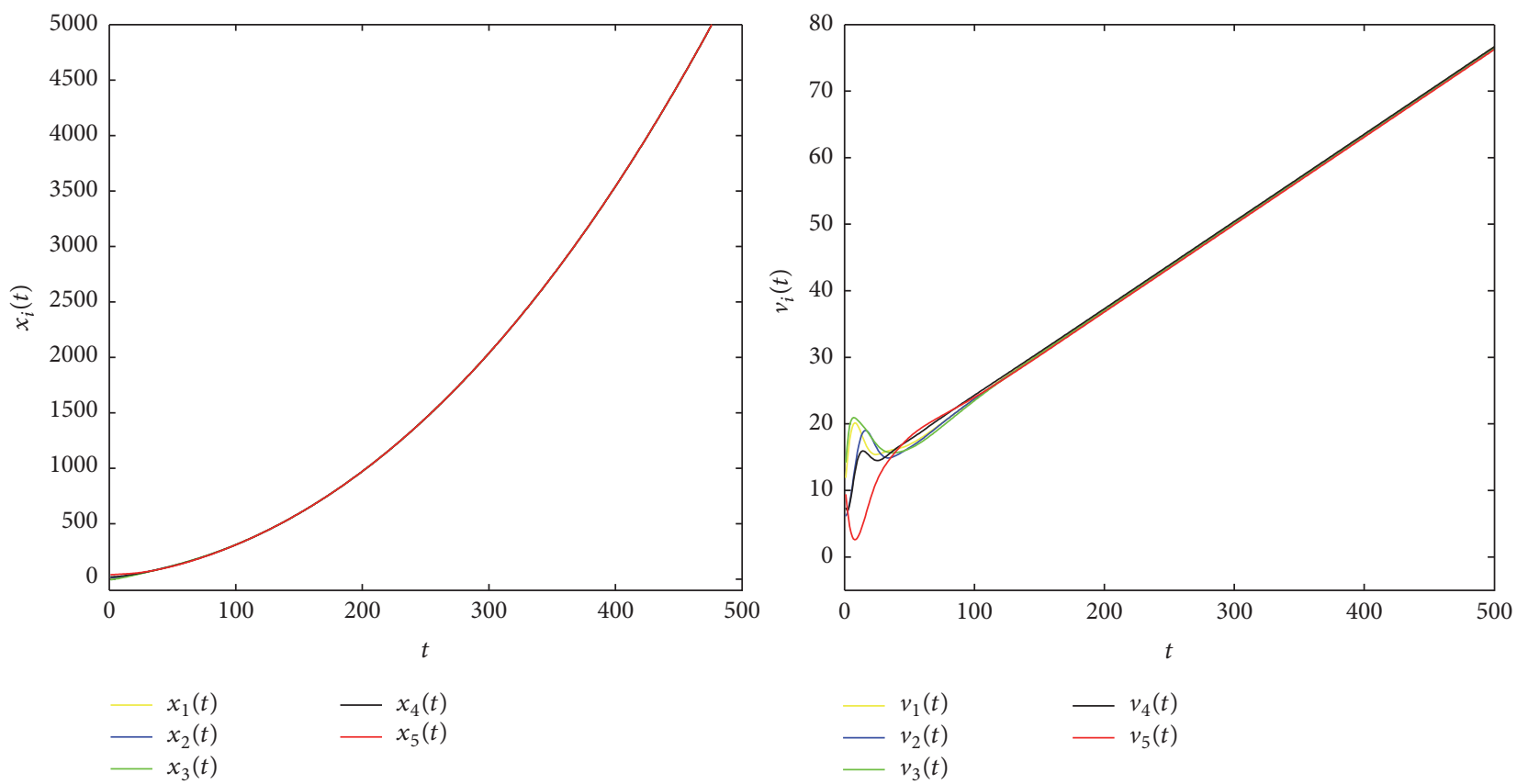

Figure 5: $x_{i}(t)$ and $v_{i}(t)$ of each agent with protocol (22), $\alpha=1.3$.

Remark 10. The simulation shows the proposed method in this paper is effective. The convergence speed with control protocol (7) is less than that with control protocol (22), but less information of neighboring agents is used in (7), which may save computation and online resources.
Remark 11. The initial states for each agent in the simulations can be chosen arbitrarily, which will not affect the asymptotic behavior of consensus. But it also should be pointed out that different initial states or different control protocol may result in different final consensus values. In other words, 
the asymptotic behavior of consensus is independent of the selections of initial states, but the final consensus values or common values are dependent on the initial states or control protocol.

\section{Conclusions}

The consensus problem for fractional-order double-integrator multiagent systems is investigated, where the fractionalorder satisfies $0<\alpha<2$. Consensus algorithms with a control parameter are designed. Based on the algebraic graph theory, Mittag-Leffler function, and Laplace transform, a necessary and sufficient condition is derived, which ensures that the fractional-order double-integrator multiagent systems can achieve consensus asymptotically. Due to the fact that communication graph of agents is always variable with time, the fractional-order multiagent systems with switching topologies will be studied in future work.

\section{Competing Interests}

The authors declare that there is no conflict of interests regarding the publication of this paper and the mentioned received funding does not lead to any conflict of interests regarding the publication.

\section{Acknowledgments}

This work is supported partly by National Natural Science Foundation of China under Grant 61673080, partly by Natural Science Foundation of Chongqing under Grant cstc2013jcyjA00026, and partly by Training Programme Foundation for the Talents of Higher Education by Chongqing Education Commission.

\section{References}

[1] X. Zhang, L. Liu, and G. Feng, "Leader-follower consensus of time-varying nonlinear multi-agent systems," Automatica, vol. 52, pp. 8-14, 2015.

[2] J. A. Fax and R. M. Murray, "Information flow and cooperative control of vehicle formations," IEEE Transactions on Automatic Control, vol. 49, no. 9, pp. 1465-1476, 2004.

[3] W. Ren and E. Atkins, "Distributed multi-vehicle coordinated control via local information exchange," International Journal of Robust and Nonlinear Control, vol. 17, no. 10-11, pp. 1002-1033, 2007.

[4] F. Cucker and S. Smale, "Emergent behavior in flocks," IEEE Transactions on Automatic Control, vol. 52, no. 5, pp. 852-862, 2007.

[5] C. Nowzari and J. Cortés, "Self-triggered coordination of robotic networks for optimal deployment," Automatica, vol. 48, no. 6, pp. 1077-1087, 2012.

[6] Y. Fan, G. Feng, Y. Wang, and C. Song, "Distributed eventtriggered control of multi-agent systems with combinational measurements," Automatica, vol. 49, no. 2, pp. 671-675, 2013.

[7] R. C. Koeller, "Toward an equation of state for solid materials with memory by use of the half-order derivative," Acta Mechanica, vol. 191, no. 3-4, pp. 125-133, 2007.
[8] J. Sabatier, O. P. Agrawal, and J. A. T. Machado, Advances in Fractional Calculus: Theoretical Developments and Applications in Physics and Engineering, Springer, Dordrecht, Netherlands, 2007.

[9] B. Kania, T. Dziwiński, W. Bauer, M. Zagórowska, and J. Baranowski, "A comparison between integer order and noninteger order controllers applied to a water levelling system," Automatics, vol. 18, no. 2, pp. 71-82, 2014.

[10] Z. Liao, C. Peng, W. Li, and Y. Wang, "Robust stability analysis for a class of fractional order systems with uncertain parameters," Journal of the Franklin Institute, vol. 348, no. 6, pp. 11011113, 2011.

[11] Y. Cao and W. Ren, "Distributed formation control for fractional-order systems: dynamic interaction and absolute/ relative damping," Systems \& Control Letters, vol. 59, no. 3-4, pp. 233-240, 2010.

[12] Y. C. Cao, Y. Li, W. Ren, and Y. Q. Chen, "Distributed coordination of networked fractional-order systems," IEEE Transactions on Systems, Man, and Cybernetics, Part B: Cybernetics, vol. 40, no. 2, pp. 362-370, 2010.

[13] Y. Cao, Y. Li, W. Ren, and Y. Q. Chen, "Distributed coordination algorithms for multiple fractional-order systems," in Proceedings of the 47th IEEE Conference on Decision and Control (CDC '08), pp. 2920-2925, Cancun, Mexico, December 2008.

[14] C. Song and J. D. Cao, "Consensus of fractional-order linear systems," in Proceedings of the 9th Asian Control Conference (ASCC '13), Istanbul, Turkey, June 2013.

[15] Z. Yu, H. Jiang, and C. Hu, "Leader-following consensus of fractional-order multi-agent systems under fixed topology," Neurocomputing, vol. 149, pp. 613-620, 2015.

[16] Z. Y. Yu, H. J. Jiang, C. Hu, and J. Yu, "Leader-following consensus of fractional-order multi-agent systems via adaptive pinning control," International Journal of Control, vol. 88, no. 9, pp. 1746-1756, 2015.

[17] H.-Y. Yang, L. Guo, Y.-L. Zhang, and X.-M. Yao, "Movement consensus of complex fractional-order multi-agent systems," Acta Automatica Sinica, vol. 40, no. 3, pp. 489-496, 2014.

[18] X. Yin, D. Yue, and S. Hu, "Consensus of fractional-order heterogeneous multi-agent systems," IET Control Theory \& Applications, vol. 7, no. 2, pp. 314-322, 2013.

[19] G. Ren, Y. Yu, and S. Zhang, "Leader-following consensus of fractional nonlinear multiagent systems," Mathematical Problems in Engineering, vol. 2015, Article ID 919757, 8 pages, 2015.

[20] C. Song, J. Cao, and Y. Liu, "Robust consensus of fractionalorder multi-agent systems with positive real uncertainty via second-order neighbors information," Neurocomputing, vol. 165, pp. 293-299, 2015.

[21] L. Chen, Y. Chai, R. Wu, and J. Yang, "Stability and stabilization of a class of nonlinear fractional-order systems with caputo derivative," IEEE Transactions on Circuits and Systems II: Express Briefs, vol. 59, no. 9, pp. 602-606, 2012.

[22] W. Ren and R. W. Beard, "Consensus seeking in multiagent systems under dynamically changing interaction topologies," IEEE Transactions on Automatic Control, vol. 50, no. 5, pp. 655661, 2005.

[23] W. Zhu and D. Z. Cheng, "Leader-following consensus of second-order agents with multiple time-varying delays," Automatica, vol. 46, no. 12, pp. 1994-1999, 2010.

[24] J. Sabatier, M. Moze, and C. Farges, "LMI stability conditions for fractional order systems," Computers \& Mathematics with Applications. An International Journal, vol. 59, no. 5, pp. 1594$1609,2010$. 
[25] M. De la Sen, "About robust stability of Caputo linear fractional dynamic systems with time delays through fixed point theory," Fixed Point Theory and Applications, vol. 2011, Article ID 86793, 19 pages, 2011.

[26] Z. Y. Lin, B. Francis, and M. Maggiore, "Necessary and sufficient graphical conditions for formation control of unicycles," IEEE Transactions on Automatic Control, vol. 50, pp. 121-127, 2005. 


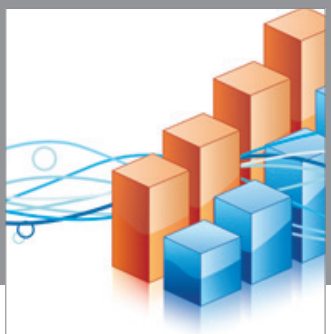

Advances in

Operations Research

vatem alat4

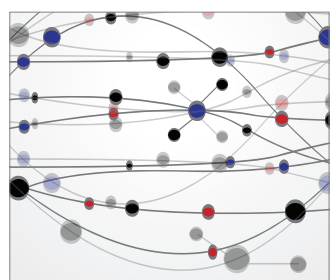

\section{The Scientific} World Journal
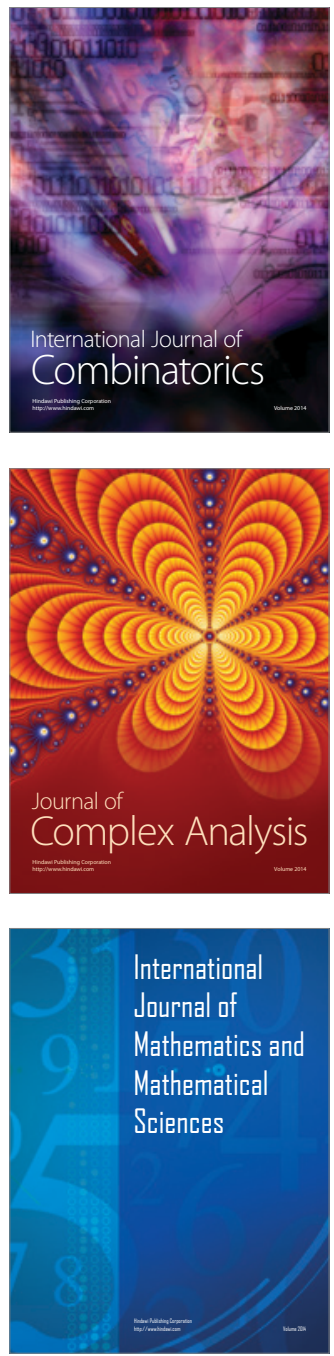
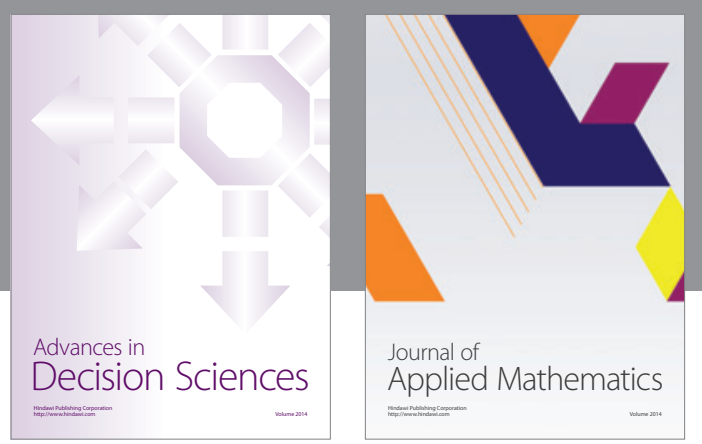

Algebra

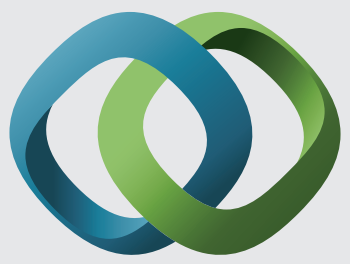

\section{Hindawi}

Submit your manuscripts at

https://www.hindawi.com
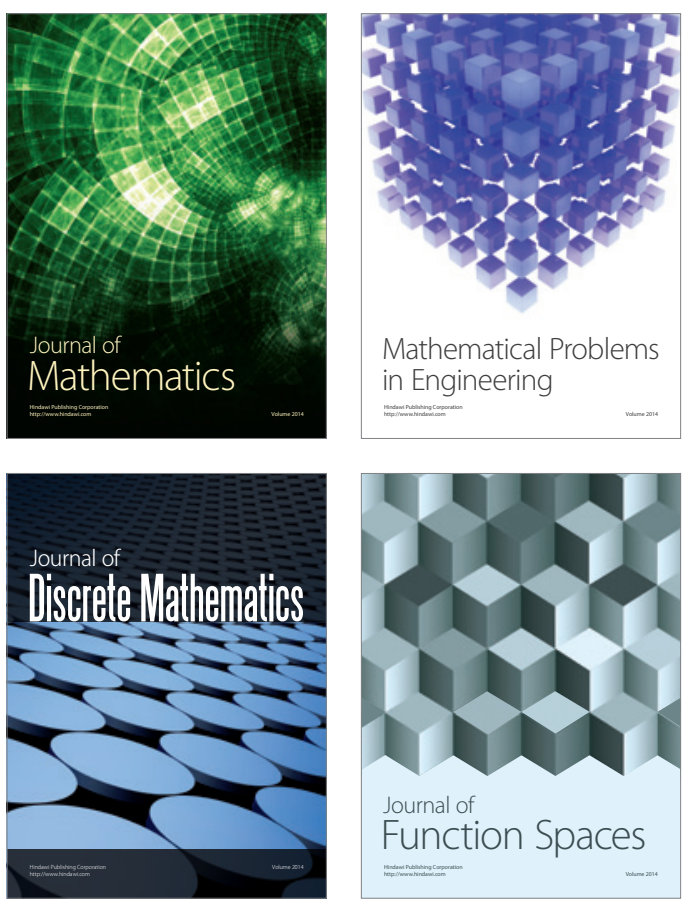

Mathematical Problems in Engineering
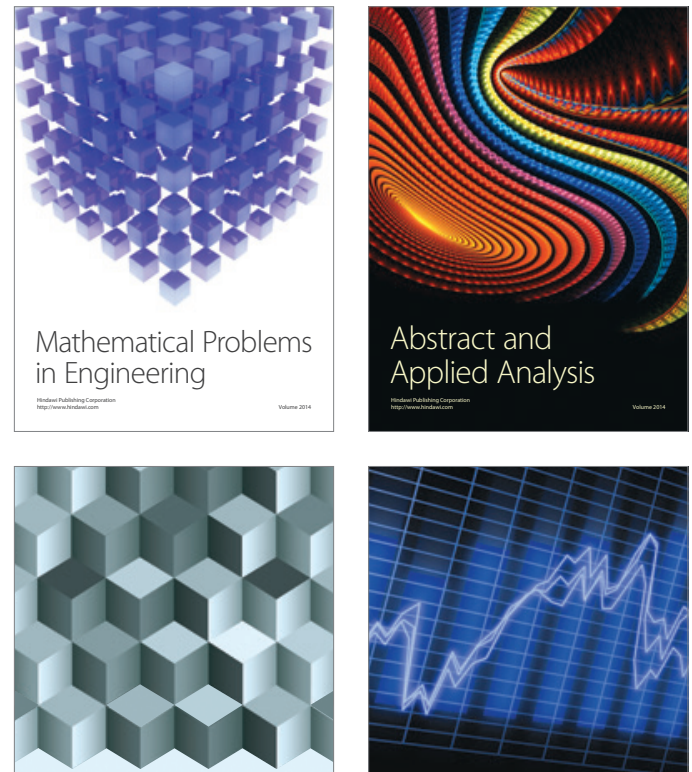

Journal of

Function Spaces

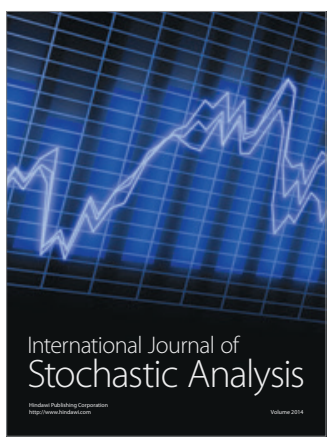

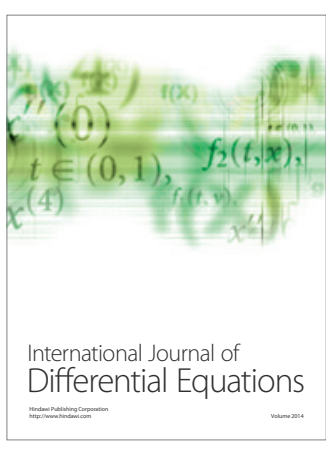
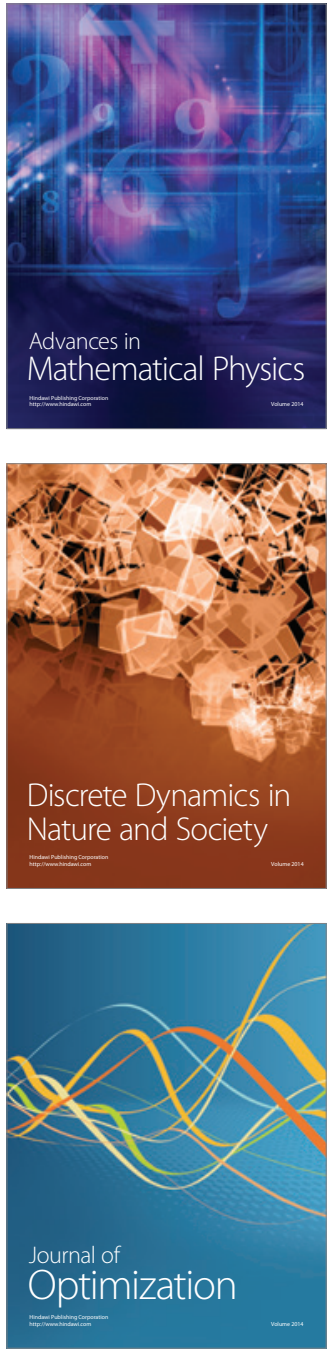